\title{
THE CALABI CONSTRUCTION FOR COMPACT RICCI FLAT RIEMANNIAN MANIFOLDS
}

\author{
BY ARTHUR E. FISCHER ${ }^{1}$ AND JOSEPH A. WOLF ${ }^{2}$
}

Communicated by S. S. Chern, July 6, 1973

1. The main result and some consequences. In $1956 \mathrm{E}$. Calabi [6] attacked the classification problem of compact euclidean space forms by means of a special construction, called the Calabi construction (see Wolf [14, p. 124]). Here we announce that the construction can be extended to compact riemannian manifolds whose Ricci curvature tensor is zero (Ricci flat). Of course, it is not known if there exist any Ricci flat nonflat compact riemannian manifolds, and in fact a search for such manifolds was the original motivation for our study. However, as a consequence of our extension of Calabi's result we reduce the question of existence of a compact nonflat Ricci flat manifold to the simply connected, connected case. In any case, we essentially reduce the construction of compact Ricci flat manifolds to the lower-dimensional case together with the case of first Betti number zero.

As a further consequence of our construction we extend one of the Bieberbach theorems [4], [14, Theorem 3.3.1] from the flat to the Ricci flat case (Theorem 1.4) and give various sufficient topological conditions for a Ricci flat manifold to be flat.

Our main result is the following:

MAIN THEOREM 1.1. Let $M^{n}$ be a compact connected Ricci flat riemannian n-manifold with first Betti number $k=b_{1}\left(M^{n}\right)$. Then there is a finite normal riemannian covering

where

$$
p: T^{k} \times M^{n-k} \rightarrow M^{n}=\Psi \backslash T^{k} \times M^{n-k}
$$

(1) $T^{k}$ is a flat riemannian $k$-torus;

(2) $\Psi=\{(h(\varphi), \varphi) \mid \varphi \in \Phi\}$ where $\Phi$ is a finite group of isometries of $M^{n-k}, h$ is an injective homomorphism of $\Phi$ into the translation group of $T^{k}$ (so $\Psi$ acts freely and properly discontinuously on $T^{k} \times M^{n-k}$ );

AMS (MOS) subject classifications (1970). Primary 53C25.

Key words and phrases. Calabi construction, Ricci flat, positive semidefinite, Ricci curvature, Betti numbers, generalized nilmanifold.

1 Research partially supported by NSF Grant GP-39060.

${ }^{2}$ Research supported by the Miller Institute for Basic Research in Science and by NSF Grant GP-16651. 
(3) $M^{n-k}$ is a compact connected Ricci fiat riemannian ( $\left.n-k\right)$-manifold which has no nonzero $\Phi$-invariant parallel vector fields.

Conversely, given $T^{k}, M^{n-k}$, and $\Psi$ as above, $M^{n}=\Psi \backslash T^{k} \times M^{n-k}$ is a compact Ricci flat connected riemannian n-manifold with first Bettinumber $k . M^{n}$ is determined up to affine equivalence by $\left(M^{n-k}, \Phi, k\right)$.

See $\S 4$ for an indication of the proof.

Thus, modulo identifications by a finite group of isometries, it is possible to split off a flat $b_{1}$-dimensional torus from a Ricci flat riemannian manifold. This reduces the affine classification of compact $n$-dimensional Ricci flat manifolds to

(i) the classification in dimensions $<n$,

(ii) the classification in dimension $n$ with $b_{1}=0$, and

(iii) the classification of finite abelian groups $\Phi$ of isometries of compact Ricci flat manifolds $M^{n-k}, 0 \leqq k<n$, such that $M^{n-k}$ has no nonzero $\Phi$-invariant parallel vector fields.

As a corollary we obtain the following result of Willmore [12], which generalizes the classical result [11] that in dimension $\leqq 3$ every Ricci flat metric is flat.

COROLLARY 1.2. Let $M$ be a compact connected n-manifold. Suppose that $b_{1}(M) \geqq n-3$, or that $M$ has a finite topological covering $\pi: \tilde{M} \rightarrow M$ with $b_{1}(\tilde{M}) \geqq n-3$. Then every Ricci flat riemannian structure on $M$ is flat.

Proof. Apply the theorem to $\tilde{M}$ with the metric pulled back from $M$. Then $\tilde{M}^{n-k}$ is a Ricci flat riemannian manifold of dimension $\leqq 3$ and so is flat.

Iterating the construction in the Main Theorem, we have

COROLLARY 1.3. Let $M^{n}$ be a compact connected Ricci flat riemannian $n$-manifold. Then there exists a series of finite normal riemannian coverings

$$
T^{k_{r}} \times M^{n-k_{r}} \rightarrow T^{k_{r-1}} \times M^{n-k_{r-1}} \rightarrow \cdots \rightarrow T^{k_{1}} \times M^{n-k_{1}} \rightarrow M^{n}
$$

where $b_{1}\left(M^{n}\right)=k_{1}<k_{2}<\cdots<k_{r}$, each $T^{k_{i}}$ is a flat riemannian $k_{i}$-torus, each $M^{n-k_{i}}$ is a compact connected Ricci flat riemannian $\left(n-k_{i}\right)$-manifold, and $b_{1}\left(M^{n-k i}\right)=k_{i+1}-k_{i}$ for $1 \leqq i<r$, and $b_{1}\left(M^{n-k_{r}}\right)=0$.

One of the Bieberbach theorems ([4]; see [14, Theorem 3.3.1]), says that a compact euclidean space form admits a finite normal riemannian covering by a flat torus. We can extend this result to the Ricci flat case as follows:

THEOREM 1.4. Let $M$ be a compact connected Ricci flat riemannian manifold. Then there is a finite normal riemannian covering $T \times N \rightarrow M$ where $T$ is a fiat riemannian torus, $\operatorname{dim} T \geqq b_{1}(M)$, and $N$ is a compact simply connected Ricci flat riemannian manifold. 
The proof follows from a result of Cheeger and Gromoll ([7, Theorem 3]) which reduces the proof to the case where $1 \rightarrow$ (finite) $\rightarrow \pi_{1}(M) \rightarrow Z^{k} \rightarrow 1$ with $k=b_{1}(M)$. In that case $\pi_{1}\left(M^{n-k}\right)$ is finite in the Main Theorem; let $N$ be its universal cover.

Thus if compact simply connected Ricci flat manifolds are flat, then compact Ricci flat manifolds are flat. Note also that if $M$ is flat, then $N$ reduces to a point so that the corollary specializes to the Bieberbach theorem for flat manifolds.

Using this extension of the Bieberbach theorem, we can find some interesting sufficient topological conditions for Ricci flat manifolds to be flat.

THEOREM 1.5. Let $M$ be a compact connected Ricci flat n-dimensional riemannian manifold. Then the following are equivalent:

1. $M$ is flat.

2. For $k>1$, the homotopy groups $\pi_{k}(M)=0$.

3. The universal covering of $M$ is acyclic.

Thus, for example, if a given compact manifold admits a flat riemannian metric it is covered by a torus, so that any Ricci flat riemannian metric is flat (see also Corollary 2.5). Thus a given compact manifold may either have flat riemannian structures, or nonflat Ricci flat riemannian structures, but not both. This remark is useful in studying some submanifolds of the space of riemannian metrics on a given compact manifold [8].

2. Riemannian manifolds with positive semidefinite Ricci curvature. We let $\boldsymbol{r}$ denote the Ricci tensor of the riemannian manifold $M$, $\chi_{\|}=\{X: \nabla X=0\}=\{$ the parallel vector fields on $M\}$, and $\mathscr{H}=\{X \mid \Delta X=0\}=$ \{the harmonic vector fields on $M\}$ where $\Delta=d \delta+\delta d$ is the Laplace-de Rham operator acting by duality on vector fields.

Using classical results of Bochner ([5], [15, pp. 37, 39, 43]), it is straightforward to prove:

THEOREM 2.1. Let $M$ be a compact connected riemannian manifold with positive semidefinite Ricci tensor and with first Betti number $b_{1}(M)$. Then $\chi_{\|}=\mathscr{H}$ and is a central ideal of dimension $b_{1}(M)$ in the Lie algebra of all Killing vector fields on $M$. Further, $\chi_{\|}$defines a foliation of $M$ by flat totally geodesic submanifolds of dimension $b_{1}(M)$.

If $M$ is Ricci flat, then $\chi_{\|}$is the Lie algebra of Killing vector fields on $M$, and $I(M)^{0}$, the identity component of the isometry group, is a torus group $T$ of dimension $b_{1}(M)$. $T$ acts effectively and smoothly on $M$, and the orbits of this action foliate $M$ by flat totally geodesic riemannian $b_{1}(M)$-tori.

If $M$ has Ricci tensor positive semidefinite, then we can extract some consequences concerning the Betti numbers $b_{p}(M)$ of $M$. 
THEOREM 2.2. Let $M$ be a compact connected n-dimensional riemannian manifold with positive semidefinite Ricci tensor and first Betti number $b_{1}(M)=k$. Then

$$
b_{p}(M) \geqq\left(\begin{array}{l}
k \\
p
\end{array}\right) \text { for } p \leqq k .
$$

Also, $b_{1}(M) \leqq n$, and $b_{1}(M)=n$ if and only if $M$ is a flat riemannian $n-t o r u s$. If $M$ is orientable, then $b_{1}(M) \neq n-1$.

In $[2, \S 8]$ and $[3, \S 8]$, Berger shows that a Ricci flat variation of a flat riemannian metric remains flat. Using Theorem 2.2 and the same technique as used in Corollary 1.2, we show that the variation is not needed:

THEOREM 2.3. Let $M$ be a compact connected $n$-dimensional manifold. Suppose that $M$ admits a finite topological covering $\pi: \tilde{M} \rightarrow M$ with $b_{1}(\tilde{M})=n$. If $g$ is a riemannian structure on $M$ with positive semidefinite Ricci tensor, then $\left(\tilde{M}, \pi^{*} g\right)$ is a flat riemannian torus and $g$ is a flat riemannian metric on $M$.

Theorem 2.3 has a stronger topological hypothesis but weaker geometrical hypothesis than Corollary 1.2. As a corollary we can strengthen the remark after Theorem 1.5.

COROLlary 2.5. If a compact manifold $M$ admits a flat riemannian structure, then every riemannian structure with Ricci tensor positive semidefinite is flat.

3. An application to generalized nilmanifolds. See $[13, \S 6]$ for the definitions of "nilmanifold" and "generalized nilmanifold". Using results from [1], from [13, §6], and from Theorem 2.2 above, we obtain the following strong extension of [13, Theorem 6.4].

THEOREM 3.1. Let $M$ be a compact riemannian manifold with positive semidefinite Ricci tensor. Suppose that the underlying differentiable manifold of $M$ is homotopy-equivalent to a compact generalized nilmanifold. Then $M$ is flat, i.e., is isometric to a compact euclidean space form. Also, the following conditions are equivalent: (i) $M$ is a nilmanifold, (ii) $\pi_{1}(M)$ is nilpotent, (iii) $M$ is a flat riemannian torus.

InDiCATION OF PROOF. A result of L. Auslander [1] allows us to reduce the proof to the following case. We have a connected simply connected nilpotent Lie group $N$, a discrete uniform subgroup $\Gamma$ of $N$, and a homotopy equivalence $f: M \rightarrow \Gamma \backslash N$. It then follows from [13, Theorem 6.4] that $M$ is diffeomorphic to a torus. Thus $b_{1}(M)=\operatorname{dim} M$, so Theorem 2.2 says that $M$ is a flat riemannian torus. 
Euclidean space forms are generalized nilmanifolds. Thus

COROLlary 3.2. Let $M$ be a compact riemannian manifold with positive semidefinite Ricci tensor. If $M$ is homotopy equivalent to a compact euclidean space form, then $M$ is flat.

4. Sketch of proof of main theorem. Let $\pi: \tilde{M}^{n} \rightarrow M^{n}$ be the universal riemannian covering. The $b_{1}\left(M^{n}\right)$-dimensional torus group (Theorem 2.1) lifts to a real vector group $R^{k}$ of ordinary translations along the euclidean factor of $\tilde{M}^{n}$. Thus $\tilde{M}^{n}=E^{k} \times \tilde{M}^{n-k}$ where $E^{k}$ is an euclidean $k$-space, $k=b_{1}(M)$, and the $R^{k}$-orbits are the $E^{k} \times\{m\}$ with $m \in \tilde{M}^{n-k}$. Express $M^{n}=\Gamma \backslash \tilde{M}^{n}$ where $\Gamma \subset \boldsymbol{I}\left(\tilde{M}^{n}\right)$. Every $\gamma \in \Gamma$ respects the splitting in the sense $\gamma=\left(\gamma_{1}, \gamma_{2}\right)$ with $\gamma_{1} \in I\left(E^{k}\right)$ and $\gamma_{2} \in I\left(\tilde{M}^{n-k}\right) . R^{k} \cap \Gamma$ is a lattice in $R^{k}$ and central in $\Gamma$, so each $\gamma_{1}$ is an ordinary translation of $E^{k}$.

Let $A=\left\{\gamma \in \Gamma: \gamma_{2}=1\right\}$ and $B=\left\{\gamma \in \Gamma: \gamma_{1}=1\right\}$. View $(A \times B) \subset \Gamma \subset$ $\left(\Gamma_{1} \times \Gamma_{2}\right)$ where $\Gamma_{i}=\left\{\gamma_{i}: \gamma \in \Gamma\right\}$. Since $\Gamma_{1}$ is abelian, one has $[\Gamma, \Gamma] \subset 1 \times \Gamma_{2}$. Arguing from $k=b_{1}(M)$, one sees that $\left(R^{k} \cap \Gamma\right) \times\left[\Gamma_{2}, \Gamma_{2}\right]$ has finite index in $\Gamma$. But $A=R^{k} \cap \Gamma$ and $\left[\Gamma_{2}, \Gamma_{2}\right] \subset B \subset \Gamma_{2}$. Now $A \times B$ is a normal subgroup of finite index in $\Gamma$. Define $T^{k}=A \backslash E^{k}, M^{n-k}=B \backslash \tilde{M}^{n-k}$ and $\Psi=\Gamma \backslash A \times B$. They turn out to have the required properties.

The converse is straightforward.

Further details of these and related results will appear in [9].

\section{REFERENCES}

1. L. Auslander, Bieberbach's theorems on space groups and discrete uniform subgroups of Lie groups, Ann. of Math. (2) 71 (1960), 579-590. MR 22 \#12161.

2. M. Berger, Sur les variétés d'Einstein compactes, Comptes Rendus de la III ${ }^{e}$ Reunion du Groupement des Mathematiciens d'Expression Latine, (Namur 1965), Librairie Universitaire, Louvain, 1966, pp. 35-55. MR 38 \#6502.

3. M. Berger and D. Ebin, Some decompositions of the space of symmetric tensors on a Riemannian manifold, J. Differential Geometry 3 (1969), 379-392. MR 42 \#993.

4. L. Bieberbach, Über die Bewegungsgruppen der Euklidischen Raüme. I, Math. Ann. 70 (1911), 297-336.

5. S. Bochner, Vector fields and Ricci curvature, Bull. Amer. Math. Soc. 52 (1946), 776-797. MR 8, 230.

6. E. Calabi, Closed, locally euclidean, 4-dimensional manifolds, Bull. Amer. Math. Soc. 63 (1957), Abstract 295, 135.

7. J. Cheeger and D. Gromoll, The splitting theorem for manifolds of non-negative Ricci curvature, J. Differential Geometry 6 (1971), 119-128.

8. A. Fischer and J. Marsden, Submanifolds of riemannian metrics with prescribed scalar curvature, Bull. Amer. Math. Soc. (to appear).

9. A. Fischer and J. A. Wolf, The structure of compact Ricci flat riemannian manifolds, (to appear).

10. E. Nelson, Tensor analysis, Princeton Univ. Press, Princeton, N.J., 1967.

11. J. A. Schouten and D. J. Struick, On some properties of general manifolds relating to Einstein's theory of gravitation, Amer. J. Math. 43 (1921), 213-216. 
12. T. J. Willmore, On compact Riemannian manifolds with zero Ricci curvature, Proc. Edinburgh Math. Soc. (2) 10 (1956), 131-133. MR 17, 783.

13. J. A. Wolf, Growth of finitely generated solvable groups and curvature of Riemannian manifolds, J. Differential Geometry 2 (1968), 421-446. MR 40 \#1939.

14. - Spaces of constant curvature, 2nd ed., J. A. Wolf, Berkeley, 1972.

15. K. Yano and S. Bochner, Curvature and Betti numbers, Ann. of Math. Studies, no. 32, Princeton Univ. Press, Princeton, N.J., 1953. MR 15, 989.

16. S. Yau, Compact flat Riemannian manifolds, J. Differential Geometry 6 (1972), 395-402.

Department of Mathematics, University of California, Santa Cruz, California 95060

Department of Mathematics, University of California, Berkeley, California 94720 